\title{
INTESTINAL STOMAS
}

\author{
M H Irving, O Hulme
}

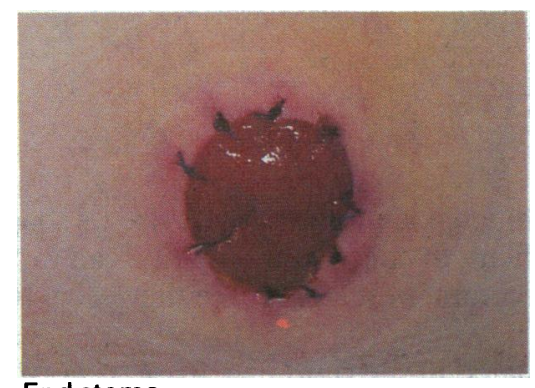

End stoma.

\section{Types of stoma}

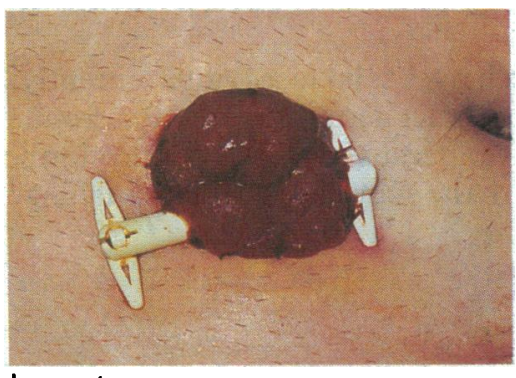

Loop stoma.

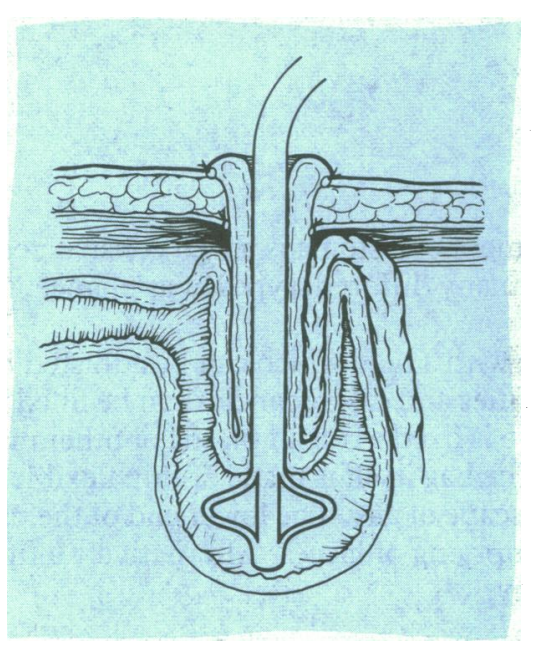

Continent stoma with catheter in pouch.

\section{Stoma care nurses}

\section{Stoma care courses}

- Hope Hospital, Salford

- North Tees General Hospital, Stockton

- The General Hospital, Birmingham.

- St Bartholomew's Hospital, London
A stoma is a surgically created opening of the bowel or urinary tract on to the body surface. The commonest procedures for producing intestinal stomas are ileostomy, colostomy, and urostomy.
There are several different types of stoma.

End stomas are the simplest to create. The divided bowel is brought through the abdominal wall and anastomosed to the skin.

Loop stomas are created when a mobile loop of bowel-of necessity either the small bowel or the mobile parts of the colon (transverse or sigmoid) - is brought through the abdominal wall and the margins sewn to the skin. Loop stomas are usually temporary, being used to protect anastomoses distal to the stoma or divert bowel contents away from diseased segments further down the bowel such as an obstructing lesion or multiple perineal fistulas. Such stomas are prone to complications and because of their bulk are difficult to manage.

Continent stomas are those in which surgical techniques are used to create a valve-like mechanism in the bowel proximal to the cutaneous opening which will allow discharge of faecal contents only when intubated. When successful such a valve avoids the need for a patient to wear an appliance.

Other ways of creating openings on the abdominal wall include caecostomy, by which the caecum is intubated with a Foley catheter, which is commonly inserted through the stump of the removed appendix. Flatus and liquid faeces can escape through the lumen of the catheter.

The distal end of a divided segment of bowel can be closed and returned to the abdomen as in the Hartmann's procedure, but it is often brought to the abdominal surface and sutured to the skin and is then termed a mucus fistula.

Although the patient's doctor will be the first to suggest that a stoma will be necessary, preoperative explanation and counselling will usually be undertaken by a stoma care nurse, who is specially trained in the management of patients with all types of stomas. Courses leading to the award of a certificate in stoma care last nine weeks and are held in four centres in the United Kingdom. 


\section{Siting the stoma}

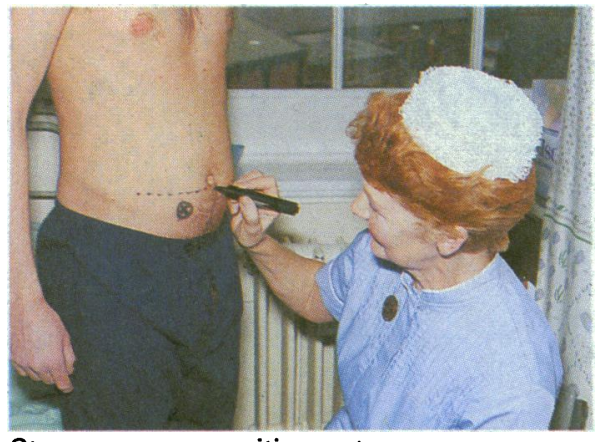

Stoma care nurse siting a stoma.

\section{Construction of the stoma}

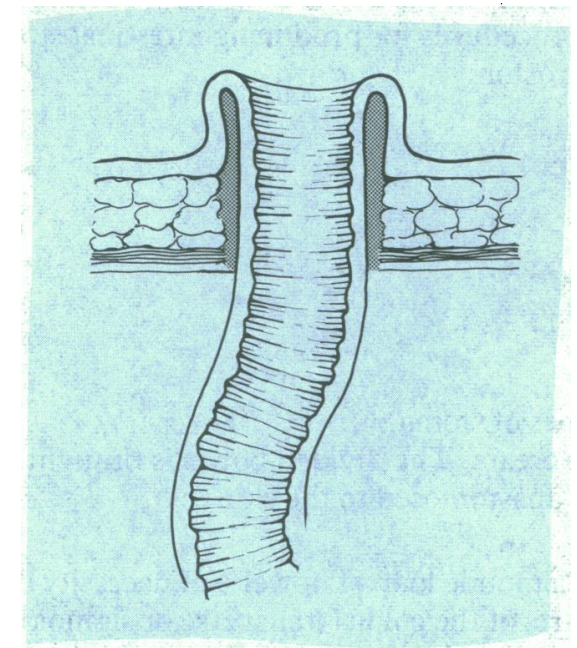

lleostomy showing eversion to produce a spout.

\section{Management}

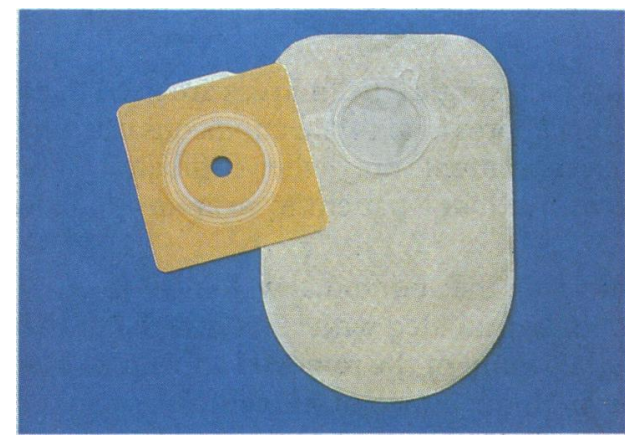

Base plate (left) is applied to skin around stoma with a clip on the bag.

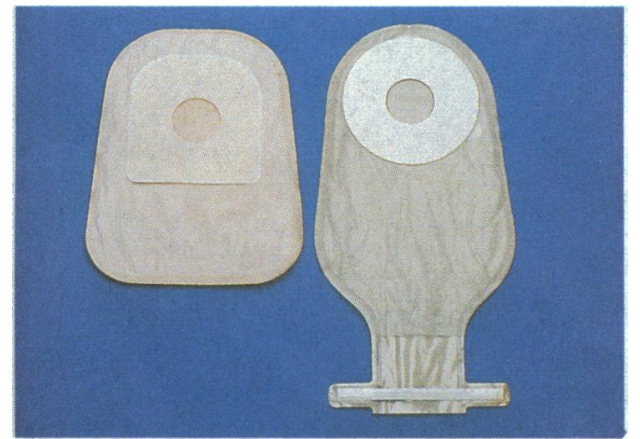

(Left) Non-drainable stoma bag. (Right) Drainable stoma bag which can be emptied without removing bag.
The presence of a stoma is enough of a burden for a patient, and the burden is increased enormously if the stoma is badly sited or badly constructed. To avoid the problem of bad siting the stoma care nurse should examine the patient before operation to determine the ideal position.

Features to be taken into account are the nature of the skin surrounding the proposed stoma-ideally it should be flat and free from scars - and the presence of anatomical irregularities such as the umbilicus and anterior superior iliac spine. Note should also be taken of clothing, such as the position of belts.

It is not only the site of the stoma that is important but also its shape. Badly constructed stomas are difficult to control and rapidly lead to deterioration in morale. The two commonest stomas are the ileal and colostomy stomas.

The end ileostomy, usually situated in the right iliac fossa, is normally constructed after panproctocolectomy for colitis. It discharges liquid faeces, which will excoriate and digest unprotected skin. For this reason the ideal ileostomy has a short spout created by everting the divided ileum back on itself. This spout ensures that the faeces can be projected into the lumen of the stoma bag.

The end colostomy usually discharges solid faeces intermittently. Although still capable of causing contact dermatitis on the surrounding skin, the faeces are not as corrosive. Because of this the stoma formed from the sigmoid colon can be flush with the skin, the solid faeces falling into the stoma bag.

\section{Appliances}

The key to successful management of a stoma is a well constructed and well fitting appliance. There are many different types of appliance, though all work on the same principle.

The base is a sheet of adhesive with a hole which can be adjusted by the patient to fit the stoma. Irregularities and discrepancies can be filled in with pastes. On to the sheet of adhesive is fixed a ring of plastic or other material, from which the bag originates. The bag itself is made of thin flexible plastic and may be vented to allow the escape of gas. The lower end of the bag may have a removable seal to allow emptying of faeces. Alternatively a full bag may be removed and thrown away.

\section{Sophisticated management}

Many patients seek to disguise the presence of a stoma or to avoid wearing a bag. Special clothing such as special swimwear is available.

Colonic stomas can be washed out by irrigating them, which, if successful, means the patient need not wear a bag.

Various occlusive devices have been developed to try to contain the faecal discharge until it is convenient to evacuate it. However, with rare exception, they have not proved effective and few patients use them. 


\section{Complications}

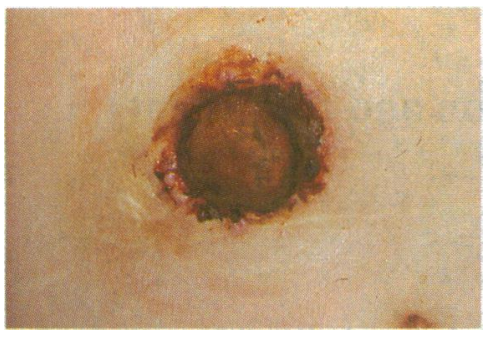

Necrotic ileostomy 48 hours after surgery. The necrotic mucosa eventually sloughed, leaving a health ileostomy.

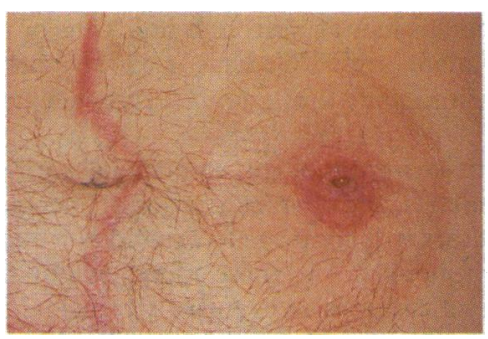

Stenosed stoma.

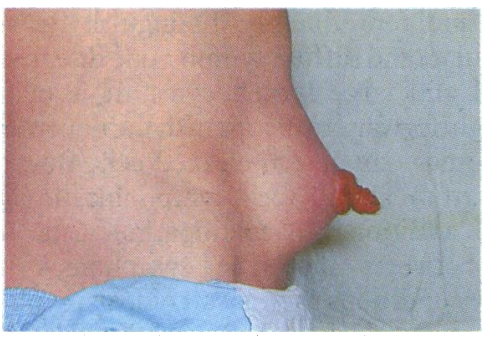

Parastomal hernia.

\section{Patient welfare}

\section{Voluntary organisations}

- British Colostomy Association, 15 Station Road

Reading,

Berkshire

- lleostomy Association,

Amblehurst Lane,

Black Scotch Lane,

Mansfield,

Nottinghamshire NG18 4PF

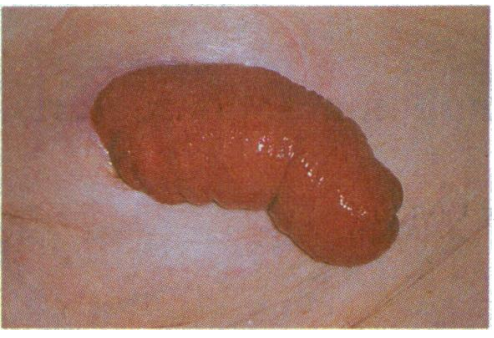

Prolapsed stoma.

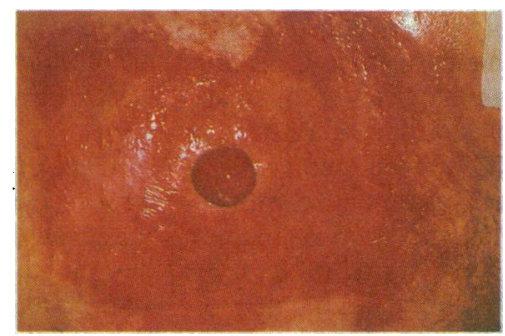

Peristomal rash.
Many problems can arise with both the structure and function of stomas. Diarrhoea and constipation can usually be controlled by drugs, but anatomical defects often need surgical correction.

Prolapse of a stoma looks alarming and is unsightly and uncomfortable but rarely is dangerous. It can be corrected by surgery.

Stenosis causes obstruction of the faecal discharge and if untreated can be serious. Local dilatation is tempting but rarely successful; it should be treated by refashioning the stoma.

Skin rashes are usually the result of failure of an appliance to fit snugly around the stoma. Occasionally they result from contact dermatitis caused by the components of the stoma bag.
Parastomal hernia is a common problem, especially in patients with colostomy. If mild it does not require any treatment. If troublesome it can commonly be controlled by a supporting belt. If severe it can be repaired by surgery.
Most patients cope well with their stoma. However, they may benefit patients with stomas. from association with one of the voluntary organisations for the welfare of

The photographs were produced by the department of medical illustration, Salford Health Authority, and the line drawings were prepared by Paul Somerset, medical illustration department, Wythenshawe Hospital.

Professor M H Irving is professor of general surgery and Mrs O Hulme is clinical nurse manager in stoma care, Hope Hospital, Salford.

The ABC of Colorectal Diseases has been edited by Mr D J Jones, lecturer and honorary senior registrar, and Professor M H Irving, department of surgery, Hope Hospital, Salford. 